\title{
Optimization of an in-house PCR method for the detection of HLA-B*27 alleles
}

\author{
NOOR AFSHAN, MUKARRAM BASHIR, HAMID NAWAZ TIPU and MOHAMMAD HUSSAIN \\ Department of Immunology, Armed Forces Institute of Pathology, 46000 Rawalpindi, Pakistan
}

Received September 20, 2017; Accepted December 18, 2017

DOI: $10.3892 / b r .2018 .1055$

\begin{abstract}
The association of spondyloarthropathies with different alleles of human leukocyte antigen (HLA) B*27 is well established. Different subtypes of HLA-B*27 may be linked with different ethnic groups, distinct clinical manifestations, specific age of onset and different prognoses. Polymerase chain reaction with sequence specific primers (PCR-SSP) is the most frequently adapted molecular method used for the recognition of HLA-B*27-specific DNA sequences. The aim of the present study was to standarise an in-house protocol of PCR-SSP for HLA-B*27 allele detection for use in the Armed Forces Institute of Pathology (AFIP), Pakistan, with consideration of its cost effectiveness. A total of 49 individual samples were included, comprising 10 transplant samples determined to be HLA-B*27-negative by PCR-SSP and 39 HLA-B*27-positive samples determined by flow cytometry, obtained from patients who were symptomatic and referred for HLA-B*27 testing. By altering each variable individually, an in-house PCR-SSP protocol was optimized to amplify common HLA-B*27 alleles (2701-2721, 2723-2730). To discriminate $\mathrm{B}^{*} 27$ from all other HLA-B alleles, a low-resolution HLA-B typing set with a 96 PCR-SSP primer mixture was used in conjunction. Among the 39 HLA-B*27-positive specimens, 31 (79\%) were detected as positive by PCR-SSP, with the remaining samples failing due to a sub-optimized protocol and/or low DNA concentration. Additionally, there was complete concordance between flow cytometry and in-house PCR, and the sensitivity and specificity of the PCR-SSP were determined to be $100 \%$. In conclusion, in-house SSP-PCR is, standard method for the detection of HLA-B*27 alleles. The determination of associations between specific HLA-B*27 alleles and AS may aid to identify individuals at higher risk of developing the disease. Furthermore, the identification of individuals at risk may aid to adapt preventive strategies.
\end{abstract}

Correspondence to: Dr Noor Afshan, Department of Immunology, Armed Forces Institute of Pathology, Harley Street, 46000 Rawalpindi, Pakistan

E-mail:afshan_ns@yahoo.com

Key words: human leukocyte antigen B27, high-resolution polymerase chain reaction, sequence specific primers, spondyloarthropathies, ankylosing spondylitis

\section{Introduction}

Ankylosing spondylitis (AS) is a progressive and chronic inflammatory disease, which primarily involves the sacroiliac joints and spine, though peripheral joints, including the shoulders, hips, ribs, heels and small joints of the hands and feet, may also be affected $(1,2)$. The most prevalent early symptoms are pain and stiffness in the affected joint, which is most severe at rest, while improving during activity (3). AS typically affects younger individuals, developing between the ages of 15 and 30. Occasionally, the disease affects multiple members of a family (4).

Class I human leukocyte antigen (HLA)-B*27 has been associated with AS in numerous studies (2). This antigen is present in over $95 \%$ of patients with AS in the Caucasian population (5-7). In the coding sequence for HLA-B*27 there is genetic polymorphism. In total, 105 polymorphism subtypes exist, namely HLA-B*2701 to HLA-B*27106, encoded by 132 alleles (8). These alleles are discriminated by changes primarily in exons 2 and 3 , which encode the $\alpha 1$ and $\alpha 2$ chains of the B27 molecule, respectively (9). HLA-B*27 typing has been proposed as a useful test for AS and related spondyloarthropathies (SpA), and thus the availability of a reliable and accurate detection method is important (3). Although the result of HLA-B*27 typing is unable confirm or exclude the diagnosis of this disease (10), different subtypes may be associated with different ethnic groups, clinical manifestation, age of onset and prognosis (11).

The microlymphocytotoxicity test (MLCT) (12) and flow cytometry (13) are commonly used techniques for routine typing of HLA-B*27. These tests rely on the detection of cell surface antigens with antibodies. Major drawbacks of MLCT include the requirement for viable cells, cross-reactivity of antigens, the requirement for a single individual to provide consistent and accurate results, and unavailability of $\mathrm{B}^{*} 27$ specific antisera covering all HLA-B*27 alleles (14). Flow cytometry is often used due to its efficiency as an alternative HLA-B*27 detection technique; however, cross-reactivity with other HLA-B molecules including B7 is a major limitation $(15,16)$. Furthermore, it requires expensive reagents, complex equipment and specifically trained personnel. As a result, many laboratories have now adopted replacement molecular methods.

The two main molecular methods most frequently adapted for histocompatibility typing are polymerase chain 
reaction with sequence specific primers (PCR-SSP) or with sequence specific oligonucleotide probes (PCR-SSO) (14). In particular, the PCR-SSP technique relies on the recognition of HLA-B*27 specific DNA sequences, and thus is an ideal test for HLA-B*27. Previous results have concluded that the outcomes obtained by PCR-SSP are more accurate compared with the conventional serological approach (15).

PCR-SSP amplification of HLA-B*27 loci is established as a precise and accurate method. It is based on the basic PCR principle that under controlled conditions, primers with complementary sequences to target sequences result in amplified products, while non-complementary primers do not initiate amplification (17). Nevertheless, an internal control primer pair, which amplifies a conserved region, is included in each PCR reaction mix in addition to sequence-specific primers, to indicate the integrity of the PCR reaction (18).

The present study focused on optimization and evaluation of in-house sequence-specific PCR for HLA-B*27 alleles. It differentiated 29 commonly occurring and disease-associated HLA-B*27 alleles $(\mathrm{B} * 2701-\mathrm{B} * 2721$ and $\mathrm{B} * 2723-\mathrm{B} * 2730)$ to the 4-digit level (19). The determination of associations between specific HLA-B*27 alleles with AS may aid to identify individuals who are likely to develop the disease, and if the population frequency of disease-associated alleles is low, then the diagnostic accuracy of the test will be increased. Furthermore, the identification of individuals at risk may aid to adapt preventive strategies.

\section{Materials and methods}

Samples. A total of 39 individual HLA-B*27-positive specimens, determined by flow cytometry (20), were obtained from patients (mean age, 31.8 \pm 8.6 years) who were symptomatic for $\mathrm{SpA}$ and referred to the Armed Forces Institute of Pathology (AFIP, Rawalpindi, Pakistan) for HLA-B*27 testing between April and December 2016. Individuals presenting with a history of trauma and/or mechanical back pain were excluded. Blood specimens from 10 HLA-B*27-negative transplant donors (mean age, $18.9 \pm 16.3$ years) were included as negative controls following HLA typing using a low-resolution HLA-B typing set consisting of 96 primer mixtures (micro SSP 1L96 wells tray; One Lamda; Thermo Fisher Scientific, Inc., Waltham, MA, USA). Table I lists the HLA-B specificities of the 10 negative controls tested. The institutional review board of AFIP approved the study and written informed consent from all patients was obtained.

In-house PCR-SSP method. Chemicals and reagents used for HLA-B*27 amplifications. Polymerase chain reaction buffer [10X with $\left(\mathrm{NH}_{4}\right)_{2} \mathrm{SO}_{4}$; Thermo Fisher Scientific, Waltham, MA, USA], a customized HLA-B*27 allele primer set of 35 (Alpha DNA, Montreal, QC, Canada) (19), dNTPs, $\mathrm{MgCl}_{2}$ (both from Thermo Fisher Scientific), HLA-DRB1 locus-specific primers (21) as internal control primer (Alpha DNA), Taq DNA polymerase (NovaTaq ${ }^{\mathrm{TM}}$ DNA Polymerase; EMD Millipore, Billerica, MA, USA), a Gentra Puregene Blood kit (Qiagen, Inc., Valencia, CA, USA), dimethyl sulfoxide (DMSO; Scharlab S.L., Barcelona, Spain) Tris base (Ultra-Pure Tris buffer; Invitrogen; Thermo Fisher Scientific), EDTA, boric acid (both from Merck KGaA, Damstadt Germany), agarose (OmniPur; EMD Millipore), bromophenol blue (Merck
Table I. HLA-B specificities of the 10 HLA-B*27-negative samples tested.

\begin{tabular}{lc}
\hline HLA-B specificity & Total samples, $\mathrm{n}$ \\
\hline $\mathrm{B} * 13$ & 1 \\
$\mathrm{~B} * 14$ & 1 \\
$\mathrm{~B} * 15$ & 2 \\
$\mathrm{~B} * 35$ & 4 \\
$\mathrm{~B} * 40$ & 3 \\
$\mathrm{~B} * 44$ & 2 \\
$\mathrm{~B} * 51$ & 4 \\
$\mathrm{~B} * 55$ & 2 \\
$\mathrm{~B} * 58$ & 1 \\
\hline
\end{tabular}

Samples were typed using a commercial kit (micro SSP 1L96 wells tray; One Lamda; Thermo Fisher Scientific, Inc., Waltham, MA, USA). HLA, human leukocyte antigen.

KGaA), gel loading dye [20\% (w/v) sucrose, $0.25 \%(w / v)$ bromophenol blue], ethidium bromide (Invitrogen; Thermo Fisher Scientific) and Mili-Q water (EvoQua, Pittsburgh, PA, USA) were used in PCR-SSP.

DNA extraction for the amplification of SSP. Venous blood samples $(3 \mathrm{ml})$ were collected in EDTA-potassium tubes from each patient. DNA was extracted using the Gentra Puregene Blood kit, according to the manufacturer's instructions. The isolated DNA was washed in ethanol and the final concentration was adjusted to $100 \mathrm{ng} / \mu \mathrm{l}$. Different concentrations of DNA (0.1-100 ng/ $\mu \mathrm{l})$ were initially tested to obtain clear bands for HLA-B*27 and to minimize nonspecific amplification. DNA purity ratio was maintained between 1.7 and 2.0, determined at 260 and $280 \mathrm{~nm}$ using an Epoch microplate spectrophotometer (BioTek Instruments, Inc., Winooski, VT, USA). DNA was stored at $-20^{\circ} \mathrm{C}$ until analysis.

PCR master mix preparation. The final optimized reaction mix was composed of the following: DNA Taq polymerase (5 U/ $\mu \mathrm{l}$ ), $0.03 \mathrm{U} / 17 \mu \mathrm{l}$ SSP reaction; dNTPs (final concentration of each dNTP, $0.172 \mathrm{mM}$ ); PCR buffer, $17 \mathrm{mM}$; Tris-HCL, $64 \mathrm{mM}, \mathrm{pH} 8.8 ; \mathrm{MgCl}_{2}, 1.7 \mathrm{mM}$; DMSO, 2\% (v/v); internal control primers (forward and reverse), $0.23 \mu \mathrm{M}$ each; and Mili-Q water, $208 \mu \mathrm{l}$. This reaction mixture was equally divided into 29 reaction tubes and one negative control per sample (15 $\mu$ l each).

Amplification control primers. The control primer pair included in master mix that amplified the third intron of the DRB1 gene (21) had the following sequences: HLA-DRB1 forward, 5'-TGCCAAGTGGAGCACCCAA-3' and reverse, 5'-GCATCTTGCTCTGTGCAGAT-3'. These primers matched conserved sequences and thus functioned as an internal positive amplification control.

Allele specific primers. A total of 35 sequence-specific primers for HLA-B*27 alleles (19) were purchased (Alpha DNA) as lyophilized, salt-purified oligonucleotides. Following reconstitution in Mili Q water, $100 \mu \mathrm{M}$ stock solution was made, 
Table II. Human leukocyte antigen $\mathrm{B} * 27$ allele primer sequences and specificities.

\begin{tabular}{|c|c|c|c|c|c|c|c|}
\hline \multicolumn{3}{|r|}{ Forward primer } & \multicolumn{3}{|r|}{ Reverse primer } & \multirow{2}{*}{$\begin{array}{l}\text { Amplicon } \\
\text { size, bp }\end{array}$} & \multirow{2}{*}{$\begin{array}{l}\mathrm{B} * 27 \text { alleles } \\
\text { amplified }\end{array}$} \\
\hline No. & Position & Sequence, 5'-3' & No. & Position & Sequence, 5'-3' & & \\
\hline 01 & 297-314 & AGAGAACCTGCGCACCGC & 17 & $411-428$ & TCGTAGGCGTCCTGGTGG & 380 & 01 \\
\hline 02 & $149-167$ & GCTACGTGGACGACACGCT & 18 & $311-330$ & GTTGTAGTAGCGGAGCGCGA & 185 & 02,30 \\
\hline 03 & 284-302 & CACAGACTGACCGAGAGGA & 17 & $411-428$ & TCGTAGGCGTCCTGGTGG & 390 & $\begin{array}{c}03,05,09,10,13 \\
14,16,17,19,28\end{array}$ \\
\hline 04 & 284-302 & CACAGACTGACCGAGAGAG & 17 & $411-428$ & TCGTAGGCGTCCTGGTGG & 390 & $\begin{array}{c}04,08,12,15,18 \\
23,25,26\end{array}$ \\
\hline 05 & $230-248$ & GCAGGAGGGGCCGGAGTT & 17 & $411-428$ & TCGTAGGCGTCCTGGTGG & 445 & 17 \\
\hline 06 & $254-272$ & ACCGGGAGACACAGATCTC & 17 & $411-428$ & TCGTAGGCGTCCTGGTGG & 420 & 18,29 \\
\hline 07 & $261-282$ & ACACAGATCTACAAGACCAAC & 17 & $411-428$ & TCGTAGGCGTCCTGGTGG & 410 & $12,16,18,23,29$ \\
\hline 08 & 294-311 & CCGAGAGAGCCTGCGGAA & 17 & $411-428$ & TCGTAGGCGTCCTGGTGG & 380 & $08,12,18,26$ \\
\hline 09 & $254-272$ & ACCGGGAGACACAGATCTG & 19 & $354-371$ & CCATACATCGTCTGCCAA & 365 & 14 \\
\hline 03 & $284-302$ & CACAGACTGACCGAGAGGA & 20 & $363-381$ & CACGTCGCAGCCGTACATG & 340 & 07 \\
\hline 10 & $262-283$ & CACAGATCTGCAAGGCCAAGG & 21 & $412-430$ & CGTCGTAGGCGTACTGGTC & 410 & 06,21 \\
\hline 11 & $262-282$ & CACAGATCTGCAAGGCCAAG & 21 & $412-430$ & CGTCGTAGGCGTACTGGTC & 410 & 06,21 \\
\hline 03 & 284-302 & CACAGACTGACCGAGAGGA & 22 & $369-385$ & GCCCCACGTCGCAGCCG & 350 & 07,19 \\
\hline 03 & 284-302 & CACAGACTGACCGAGAGGA & 23 & $418-434$ & CTTGCCGTCGTAGGCGTG & 395 & 09 \\
\hline 03 & 284-302 & CACAGACTGACCGAGAGGA & 24 & $418-434$ & CTTGCCGTCGTAGGCGTC & 395 & $\begin{array}{c}03,05,10,13,14 \\
16,17,19,28,29\end{array}$ \\
\hline 03 & 284-302 & CACAGACTGACCGAGAGGA & 25 & $527-544$ & CTCTCAGCTGCTCCGCCT & 505 & 10 \\
\hline 03 & 284-302 & CACAGACTGACCGAGAGGA & 26 & $418-435$ & GTTTGCCGTCGTAGGCGTA & 395 & 07,27 \\
\hline 10 & $262-283$ & CACAGATCTGCAAGGCCAAGG & 20 & $363-381$ & CACGTCGCAGCCGTACATG & 360 & $07,11,24$ \\
\hline 12 & $4-14$ & CGAGATGCGGGTCACGGC & 27 & $97-114$ & CCGGGACACGGAGGTGTG & 255 & $\begin{array}{c}01-12,14-21 \\
23-30\end{array}$ \\
\hline 13 & $4-14$ & CGAGATGCGGGTCACGGA & 27 & $97-114$ & CCGGGACACGGAGGTGTG & 255 & 13 \\
\hline 14 & $83-97$ & CCACTCCATGAGGTATTTCC & 28 & $247-265$ & GTGTCTCCCGGTCCCAATG & 190 & 03 \\
\hline 14 & $83-97$ & CCACTCCATGAGGTATTTCC & 29 & $247-265$ & GTGTCTCCCGGTCCCAATA & 190 & $\begin{array}{c}01,02,04, \\
18-21,24-30\end{array}$ \\
\hline 02 & $149-167$ & GCTACGTGGACGACACGCT & 25 & $527-544$ & CTCTCAGCTGCTCCGCCT & 640 & $\begin{array}{c}04,06,10,15, \\
18,20,21,24,25\end{array}$ \\
\hline \multirow[t]{2}{*}{02} & $149-167$ & GCTACGTGGACGACACGCT & 30,31 & $280-298$ & CTCGGTCAGTCTGTGCCTT & 155 & $01-11,13-15,17$ \\
\hline & & & & 280-299 & TCTCGGTAAGTCTGTGCCTT & & $\begin{array}{c}19-21,24,25, \\
27,28,30\end{array}$ \\
\hline 02 & $149-167$ & GCTACGTGGACGACACGCT & 32 & $559-576$ & GAGCCACTCCACGCACGT & 675 & 15,28 \\
\hline 16 & $344-363$ & GGTCTCACACCCTCCAGAAT & 33 & $559-576$ & GAGCCACTCCACGCACAG & 235 & 25 \\
\hline 15 & $302-319$ & CCTGCGGACCCTGCTCC & 34 & $363-383$ & CACGTCGCAGCCGTACATC & 325 & 19,21 \\
\hline 03 & $284-302$ & CACAGACTGACCGAGAGGA & 32 & $559-576$ & GAGCCACTCCACGCACGT & 540 & 28 \\
\hline 09 & $254-272$ & ACCGGGAGACACAGATCTG & 35 & $363-383$ & CACGTCGCAGCCGTACATC & 370 & $19,21,30$ \\
\hline
\end{tabular}

followed by a working dilution of $3 \mathrm{mM}$. Sequences, annealing position and specificities are listed in Table II. The primers were added to the PCR reaction tubes (29 tubes/sample) at a final concentration of $0.23 \mu \mathrm{M}$. Thus, 29 SSP mixtures were made to assign $29 \mathrm{HLA}-\mathrm{B} * 27$ alleles at the 4-digit level (B*2701-B*2721 and $\mathrm{B} * 2723-\mathrm{B} * 2730)$.

PCR protocol. Forward and reversed primers (1 $\mu \mathrm{l}$ each) were dispensed in 29 mini Eppendorf tubes according to the primer mix combinations (Table II). A total of $15 \mu 1$ master mix was added to each and to the negative control tube. PCR was performed on an Eppendorf Mastercycler Gradient thermocycler (7 tests were also run on a MultiGene OptiMax Labnet International Thermal Cycler) at an initial denaturation temperature of $94^{\circ} \mathrm{C}$ for $5 \mathrm{~min}$, followed by 30 cycles of amplification $\left(30 \mathrm{sec}\right.$ at $95^{\circ} \mathrm{C}, 60 \mathrm{sec}$ at $64^{\circ} \mathrm{C}$ and $72^{\circ} \mathrm{C}$ for $\left.60 \mathrm{sec}\right)$ and a final elongation step at $72^{\circ} \mathrm{C}$ for $7 \mathrm{~min}$, with storage at $4^{\circ} \mathrm{C}$. A total of 10 random samples were repeated.

Visualization of amplifications by agarose gel electrophoresis. Agarose gel 2\% (w/v) in 0.1X Tris/borate/EDTA (TBE) buffer was prepared. The agarose was dissolved by boiling, then cooled to $60^{\circ} \mathrm{C}$. Ethidium bromide $(0.5 \mu \mathrm{g} / \mathrm{ml}$ gel solution) was then added. A 4-5-mm thick gel with 3-mm wide slots was cast 
Table III. Positive detection of human leukocyte antigen B*27 by in-house polymerase chain reaction with sequence-specific primers.

\begin{tabular}{lc}
\hline $\begin{array}{l}\text { SpA } \\
\text { patient ID }\end{array}$ & Possible B*27 alleles \\
\hline 1 & $04,08,12,15,16,18,19,21,23,25,26,29$ \\
2 & 19,21 \\
3 & $03,05,10,13,14,16,17,19,28,29$ \\
4 & $01,02,04,18-21,24-30$ \\
5 & $01,02,04,18-21,24-30$ \\
6 & $12,16,18,19,21,23,29,30$ \\
7 & 19 \\
8 & $02,12,16,18,19,21,23,29,30$ \\
9 & 30 \\
10 & 07,19 \\
11 & $06,07,11,21,24$ \\
12 & 07 \\
13 & 06,21 \\
14 & $06,21,25$ \\
15 & 03 \\
16 & 07 \\
17 & 21 \\
18 & $07,10,19$ \\
19 & 10 \\
20 & 10 \\
21 & 07 \\
22 & $05,10,13,14,16,17,19,28,29$ \\
\hline
\end{tabular}

Suspected

SpA

patient ID

Possible B*27 alleles

$\begin{array}{lc}23 & 01,03,05,06,07,10,13,14,16,17,19,21,28,29 \\ 24 & 03,05,07,10,11,13,14,16,17,19,24,2829 \\ 25 & 07,19,21 \\ 26 & 14,19,21,30 \\ 27 & 19,21,30 \\ 28 & 07,19 \\ 29 & 07,11,24 \\ 30 & 07,19,21 \\ 31 & 01-11,13-15,17,19-21,24,25,27,28,30\end{array}$

SpA, spondyloarthropathy.

and set for 10-20 min. Following the addition of $1 \mu \mathrm{l}$ loading dye to each PCR tube, the products were loaded on the gel. The gel was run in $0.1 \mathrm{X}$ TBE buffer for $35 \mathrm{~min}$ at $4 \mathrm{~V} / \mathrm{cm}$. Finally, the gel was examined under ultra-violet illumination and the results were documented (Fig. 1).

Analytical specificity of the in-house method. The specificity of the in-house method was ensured by the selection of the primers, as well as by concurrent use of the micro SSP 1L96 wells typing tray, used to differentiate B27 from all other HLA-B alleles.
Table IV. Comparison of flow cytometry and PCR-SSP.

\begin{tabular}{lccc}
\hline In-house PCR-SSP & $\begin{array}{c}\text { Flow cytometry } \\
\text { Positive }\end{array}$ & $\begin{array}{c}\text { Low-resolution } \\
\text { PCR-SSP (kit) } \\
\text { Negative }\end{array}$ & Total \\
\hline Positive & 31 & 0 & 31 \\
Negative & 0 & 10 & 10 \\
Total & 31 & 10 & 41 \\
\hline
\end{tabular}

PCR-SSP, polymerase chain reaction with sequence specific primers.

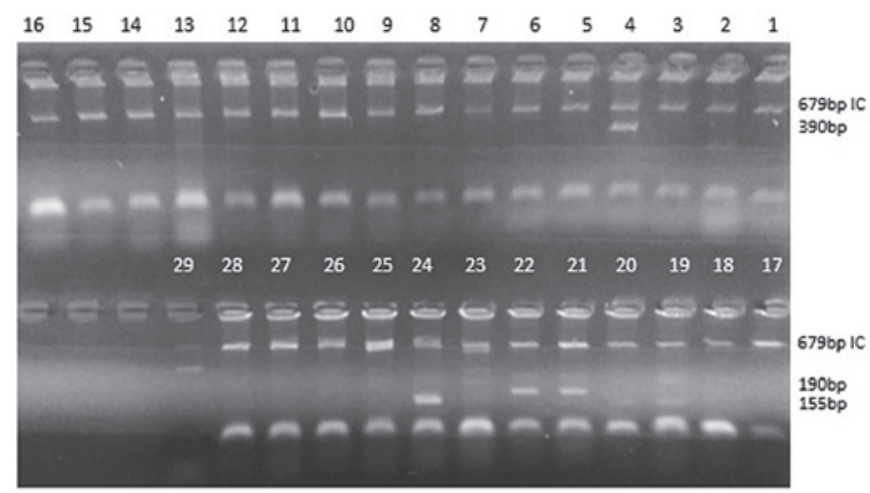

Figure 1. Agarose gel electrophoresis of positive HLA-B*27 alleles. The internal control band $(679 \mathrm{bp})$ is positive in all lanes excluding lane 29. HLA-B*27 allele bands are present in lanes 4 (390 bp), 21 (190 bp), 22 (190 bp) and 24 (155 bp). Diffuse bands at the bottom of each lane are primer dimers. HLA, human leukocyte antigen; IC, internal control.

\section{Results}

HLA-B*27 typing. A total of 189 suspected cases of SpA were referred to AFIP in 2016 for HLA-B*27 typing by flow cytometry, and 45 were identified as HLA-B*27-positive (23.8\%). In the present study, a total of 49 specimens with complete medical records were assessed: 39 HLA-B*27-positive specimens determined by flow cytometry and 10 negative donor specimens that were HLA-B*27-negative, determined by PCR-SSP.

In house PCR-SSP. Of the 39 positive samples, 31 samples exhibited positive HLA-B*27 allele results with positive internal control, listed in Table III (including 22 confirmed SpA cases, of which 20 were AS, with diagnosis confirmed on positive HLA-B*27 typing), while the remaining 8 failed (low DNA concentration) or became invalid (no internal control positive) due to sub optimized conditions. The DRB1 internal control (product size 796 bp), detected in 31 samples, confirmed the correct PCR conditions and the presence of sufficient template DNA in these amplifications. The 10 transplant donors who were HLA-B*27-negative according to the low-resolution PCR-SSP kit were also detected as negative by in-house PCR-SSP. Thus, overall sensitivity and specificity were $100 \%$, and there were no false positive or false negative results (Table IV). Similar results were obtained for 7 samples, which were run on two distinctive thermal cyclers (Eppendorf Master cycler gradient and MultiGene OptiMax 
Labnet International). In addition, 10 random samples were tested twice for HLA-B*27 using the in-house PCR-SSP test and demonstrated reproducible results.

Analytical sensitivity. Analytical sensitivity of the PCR-SSP technique was tested with different concentrations of DNA ranging from 0.1 to $100 \mathrm{ng} / \mu \mathrm{l}$. Positive bands of the control and $\mathrm{B} * 27$-specific primers were most obviously detected at concentrations ranging from 50 to $92 \mathrm{ng} / \mu \mathrm{l}$.

\section{Discussion}

The association between AS and HLA-B*27 alleles depends on ethnicity and geographical location. HLA-B*27 is more frequent in Punjabi and Urdu-speaking communities in Pakistan (22). Certain alleles exhibit an association with increased susceptibility to AS, including B*2702, B*2704, $\mathrm{B} * 2705, \mathrm{~B} * 2707$ and $\mathrm{B} * 2708(23)$, while others may confer protection, including B*2706 and B*2709 (24). Thus, subtype identification may be helpful for early diagnosis and improved prognosis of AS.

A PCR detection method for HLA-class I and II genes has been used by us for tissue typing of transplant patients, and was used as a starting point in the present study. PCR-SSP for HLA-B*27 detection is a method based on the use of specific primers complementary to unique HLA-B*27 gene sequences. In the present study, an in-house PCR-SSP test was developed which amplified common HLA-B*27 alleles (2701-2721 and 2723-2730) (19).

The specificity of PCR is influenced by numerous factors, including the sequence of the primer (GC content), ratio of primer, buffer, $\mathrm{Mg}^{2+}$ ion concentration and Taq polymerase concentration $(25,26)$. All conditions were optimized in the present study for all primer pairs in succession. This optimization resulted in a slightly different protocol from tissue typing; the primer concentration was lower and the optimal annealing temperature was higher. However, the results may contradict as when primer concentration is lower, more cycles and higher annealing temperature are required (26). The lower primer concentration was important to prevent primer dimerization. Initially in preliminary assays, $0.47 \mu \mathrm{mol} / \mu \mathrm{l}$ primer was used in each reaction, which was identified to create primerdimers (data not shown). Reducing primer concentration to $0.23 \mu \mathrm{mol} / \mu \mathrm{l}$ minimized the presence of these dimers. Another important variable in PCR is the $\mathrm{MgCl}_{2}$ concentration; an optimal concentration of $1.7 \mathrm{mM}$ was achieved in the present study by titration. Furthermore, the annealing temperature required optimization, which was increased to $64^{\circ} \mathrm{C}$, resulting in a higher specificity of synthesis of HLA-B* 27 products. The DRB1 product also exhibited improved intensity with higher annealing temperature. Therefore, an optimized annealing temperature was important to avoid false positive bands, which may otherwise lead to misdiagnosis of patients. DMSO may have also improved the PCR reaction, by decreasing inter- and intra-strand reannealing (27-29). Additionally, precautionary measures were taken to prevent contamination of samples using dedicated equipment and reagents.

The analytical sensitivity of the PCR-SSP technique was tested with different concentrations of DNA ranging from 0.1 to $100 \mathrm{ng} / \mu \mathrm{l}$. Positive bands of the control and $\mathrm{B}^{*} 27$-specific primers were most obviously detected at concentrations ranging from 50 to $92 \mathrm{ng} / \mu \mathrm{l}$. High analytical sensitivity of in-house PCR-SSP has advantages over other methods, as it requires lower quantity blood samples compared with MLCT, and older samples may be used compared with flow cytometry, which requires fresh samples for accurate results.

The present study represents an advancement in biomedical science in Pakistan, as to the best of our knowledge, it is a pioneer attempt in this region, although this work has been conducted in other countries. Immunological research is comparatively novel in Pakistan and there are few available molecular immunology laboratories. The association of different HLAs with autoimmune diseases is now an active area of research in the region.

The cost of the present in-house, optimized PCR SSP technique was relatively low; the technique was $\sim 2$ times less costly than that of imported commercial PCR-SSP test kits provided by certain laboratories. Thus, in developing countries including Pakistan, in-house protocols provide a more economical alternative. Nevertheless, applications of the present study may lead to improved understanding and treatment of general SpA diseases. Furthermore, it may form the basis for the future studies on the association of HLA-B*27 alleles with AS and related arthropathies.

In conclusion, in-house PCR-SSP should be considered a standard method for the detection of HLA-B*27 alleles. It is a relatively accurate method that bypasses the typical problems associated with MLCT and flow cytometry, and may improve the diagnosis of AS at the molecular level. Furthermore, although the technique is labour intensive, it is markedly less costly than imported commercial PCR-SSP test kits.

\section{References}

1. Ahsan T, Erum U, Jabeen R and Khowaja D: Ankylosing Spondylitis: A rheumatology clinic experience. Pak J Med Sci 32: 365-368, 2016.

2. Hospital TO: The pathogenesis of ankylosing spondylitis. Neurosurg Focus 24: 1-10, 2008.

3. Raychaudhuri SP and Deodhar A: The classification and diagnostic criteria of ankylosing spondylitis. J Autoimmun 48-49: 128-133, 2014.

4. Reveille JD: Major histocompatibility genes and ankylosing spondylitis. Best Pract Res Clin Rheumatol 20: 601-609, 2006.

5. Brown MA: Breakthroughs in genetic studies of ankylosing spondylitis. Rheumatology (Oxford) 47: 132-137, 2008.

6. Sheehan NJ and Frcp M: The ramifications of HLA-B27. J R Soc Med 97: 10-14, 2004.

7. Reveille JD, Ball EJ and Khan MA: HLA-B27 and genetic predisposing factors in spondyloarthropathies. Curr Opin Rheumatol 13: 265-272, 2001.

8. Khan MA: Polymorphism of HLA-B27: 105 subtypes currently known. Curr Rheumatol Rep 15: 1-6, 2013.

9. Tipu HN: Journal of Genetic Disorders and Genetic Reports Mini Review: HLA B27 and its immunogenetics in ankylosing spondylitis. J Genet Disor Genet Rep 2: 1, 2013.

10. Khan MA and Khan MK: Diagnostic value of HLA-B27 testing ankylosing spondylitis and Reiter's syndrome. Ann Intern Med 96: 70-76, 1982 .

11. Mou Y, Zhang P, Li Q, Lin Z, Liao Z, Wei Q and Gu J: Clinical features in Juvenile-Onset ankylosing spondylitis patients carrying different B27 subtypes. Biomed Res Int 2015: 594878, 2015.

12. Dunky A, Neumüller J, Hübner C, Fischer GF, Bayer PM, Wagner E, Schwartz DW and Mayr WR: HLA-B27 determination using serological methods. A comparison of enzyme immunoassay and a microlymphocytotoxic test with flow cytometry and a molecular biological assay. Rheumatol Int 16: 95-100, 1996. 
13. Skalska U, Kozakiewicz A, Maśliński W and Jurkowska M: HLA-B27 detection - comparison of genetic sequence-based method and flow cytometry assay. Reumatologia 53: 74-78, 2015.

14. Middelton D: HLA typing from serology to sequencing Era. Iran J Allergy Asthma Immunol 4: 53-66, 2005.

15. Seipp MT, Erali M, Wies RL and Wittwer C: HLA-B27 typing: Evaluation of an allele-specific PCR melting assay and two flow cytometric antigen assays. Cytometry B Clin Cytom 63: 10-15, 2005.

16. Cianga P, Zlei M, Rezus E and Cianga C: The flow cytometric labeling pattern in HLA-B27 detection may suggest cross reactivities. Rev Romana Med Lab 19: 185-191, 2011.

17. Chatterjee A, Sinha SK and Mukherjee G: A comprehensive review on HLA and its detections by polymerase chain reaction technique. Int J Pharm Res Sch 3: 340-346, 2014.

18. Shyamala V and Ferro-Luzzi Ames G: Single specific primerpolymerase chain reaction (SSP-PCR) and genome walking. Methods Mol Biol 15: 339-348, 1993.

19. Downing J, Coates E, Street J, Hammond L, Rees TJ, Pepperall J and Darke C: A high-resolution polymerase chain reaction-sequence-specific primer HLA-B*27 typing set and its application in routine HLA-B27 testing. Genet Test 10: 98-103, 2006.

20. Hücre A, Hla B, Testinin T and Yorumu K: Accurate and simple interpretation of HLA B27 screening by flow cytometry. Turk J Immunol 4: 1-6, 2016.

21. Olerup $\mathrm{O}$ and Zetterquist H: HLA-DR typing by PCR amplification with sequence-specific primers (PCR-SSP) in 2 hours: An alternative to serological DR typing in clinical practice including donor-recipient matching in cadaveric transplantation. Tissue Antigens 39: 225-235, 1992.
22. Zafar N, Khan S, Qadir A, Raza Y, Naqvi A and Rizvi A: Hla frequencies in Pakistani population. JPMA 46: 12-13, 1996.

23. Mou Y, Wu Z, Gu J, Liao Z, Lin Z, Wei Q, Huang J and Li Q HLA-B27 polymorphism in patients with juvenile and adult-onset ankylosing spondylitis in Southern China. Tissue Antigens 75: 56-60, 2010.

24. Cheng X, Mei Y, Ji X, Xue Q and Chen D: Molecular mechanism of the susceptibility difference between HLA-B*27:02/04/05 and HLA-B*27:06/09 to ankylosing spondylitis: substitution analysis, MD simulation, QSAR modelling, and in vitro assay. SAR QSAR Environ Res 27: 409-425, 2016.

25. Sharma N, Singh P, Nautiyal SC, Shuaib M, Rawat $P$ and Kaur G: Rapid detection of HLA-B27 sequence specific alleles by in-house optimized molecular assay for the detection of ankylosing spondylitis. J Biomed Pharm Res 2: 175-180, 2013.

26. Yang R, Zhang JH and Yuan GY: Establishment of an optimized PCR method using sequence-specific primers for screening multiple gene polymorphisms simultaneously. Mol Med Rep 7: 201-204, 2013.

27. Hardjasa A, Ling M, Ma K and $\mathrm{Yu} \mathrm{H}$ : Investigating the effects of DMSO on PCR fidelity using a restriction digest-based method. J Exp Microbiol Immunol 14: 161-164, 2010.

28. Hung T, Mak K and Fong K: A specificity enhancer for polymerase chain reaction. Nucleic Acids Res 18: 4953, 1990.

29. Ralser M, Querfurth R, Warnatz HJ, Lehrach H, Yaspo ML and Krobitsch S: An efficient and economic enhancer mix for PCR. Biochem Biophys Res Commun 347: 747-751, 2006. 